\title{
Qualidade de sementes e a plasticidade da soja com diferentes hábitos de crescimento
}

\author{
Seed quality and soybean plasticity with different growth habits \\ Calidad de semillas y plasticidad de la soja con diferentes hábitos de crecimiento
}

Recebido: 04/02/2022 | Revisado: 14/02/2022 | Aceito: 22/02/2022 | Publicado: 03/03/2022

Leticia Decarli
ORCID: https://orcid.org/0000-0002-7522-5816
Instituto Federal de Educação, Ciência e Tecnologia Rio Grande do Sul, Brasil
E-mail: leticia.decarli95@gmail.com
Marcos Paulo Ludwig
ORCID: https://orcid.org/0000-0002-1350-0181
E-mail: marcos.ludwig@ibiruba.ifrs.edu.br
Júlia Decarli
Instituto Federal de Educação, Ciência e Tecnologia Rio Grande do Sul, Brasil
ORCID: https://orcid.org/0000-0001-5728-1075
Cooperativa Agroindustrial, CAPAL, Brasil
E-mail: julia.decarli90@gmail.com
Thales Garbin
ORCID: https://orcid.org/0000-0002-8390-5790
Cooperativa Agroindustrial, CAPAL, Brasil
E-mail: garbintharles@gmail.com
Juliano Dalcin Martins
ORCID: https://orcid.org/0000-0003-2501-1067
Universidade Federal de Santa Maria, Brasil
E-mail: julianodalcinmartins@gmail.com
Bruna de Villa
ORCID: https://orcid.org/0000-0002-2401-7312
Universidade Federal de Santa Maria, Brasil
E-mail: bruna.devilla.58@hotmail.com
Luciene Kazue Tokura
ORCID: https://orcid.org/0000-0001-9758-0141
Universidade Estadual do Oeste do Paraná, Brasil
E-mail: lucienetokura@gmail.com
Mateus Pereira Cerveira
ORCID: https://orcid.org/0000-0002-0266-5490
Universidade Federal de Santa Maria, Brasil
E-mail: mateus_cerveira@icloud.com

\section{Resumo}

Este trabalho teve por objetivo foi avaliar o efeito da qualidade fisiológica de sementes no plastocrono, produtividade e comportamento de cultivares de soja. O experimento foi conduzido nos períodos de 2015/2016 e 2016/2017 no planalto médio do estado do Rio Grande do Sul. Foram utilizadas cultivares determinadas (BMX Ativa RR e FPS Urano RR) e indeterminadas (5855 RSF IPRO e Don Mario 5958 RSF IPRO). Em cada ano de cultivo foram conduzidos dois ensaios em população de plantas e em plantas isoladas provenientes de sementes com qualidade superior e inferior. Foi avaliado o plastocrono durante o desenvolvimento das plantas e ao final realizada a avaliação da produtividade e dos componentes da produção. Os resultados foram avaliados por meio da análise de variância e os valores médios foram comparados pelo teste de Tukey a 5\%. O plastocrono foi influenciado pela disponibilidade hídrica, a forma de crescimento não foi determinante na definição do plastocrono. Plantas originadas de sementes de menor qualidade necessitam de maior plastocrono durante seu ciclo. Plantas de origem de sementes de qualidade superior produzem mais grãos tanto em população de plantas como de forma isolada. O desenvolvimento das plantas não foi fator determinante para a produtividade.

Palavras-chave: Glycine $\max$ (L.) Merril; Vigor; Plasticidade; Soma térmica.

\begin{abstract}
This study aimed to evaluate the effect of the physiological quality of seeds on plastocrono, productivity and behavior of soybean cultivars. The experiment was conducted in the periods 2015/2016 and 2016/2017 in the middle plateau of the state of Rio Grande do Sul. Determined (BMX Active RR and Uranus RR FPS) and undetermined (5855 RSF IPRO and Don Mario 5958 RSF IPRO) cultivars were used. In each year of cultivation were conducted two trials in plant population and isolated plants from seeds with superior and inferior quality. The plastocrono was evaluated during the development of the plants and, at the end, the evaluation of productivity and production components was performed. The
\end{abstract}


results were evaluated by analysis of variance and the mean values were compared by the Tukey test at $5 \%$. The plastocrono was influenced by the availability of water, the form of growth was not determinant in the definition of plastocrono. Plants originated from seeds of lower quality need more plastocrono during its cycle. Plants of origin of seeds of superior quality produce more grains both in plant population and in isolation. Plant development was not a determining factor for productivity.

Keywords: Glycine max (L.) Merril; Vigor; Plasticity; Thermal sum.

\section{Resumen}

Este trabajo tuvo por objetivo evaluar el efecto de la calidad fisiológica de semillas en el plastocrono, productividad y comportamiento de cultivares de soja. El experimento se llevó a cabo en los períodos 2015/2016 y 2016/2017 en la meseta media del estado de Rio Grande do Sul. Se utilizaron variedades determinadas (BMX Activa RR y FPS Urano RR) e indeterminadas (5855 RSF IPRO y Don Mario 5958 RSF IPRO). En cada año de cultivo se realizaron dos ensayos en población de plantas y en plantas aisladas provenientes de semillas con calidad superior e inferior. Se evaluó el plastocrono durante el desarrollo de las plantas y al final se realizó la evaluación de la productividad y de los componentes de la producción. Los resultados fueron evaluados por medio del análisis de varianza y los valores medios fueron comparados por el test de Tukey al 5\%. El plastocrono fue influenciado por la disponibilidad hídrica, la forma de crecimiento no fue determinante en la definición del plastocrono. Plantas originadas de semillas de menor calidad necesitan de mayor plastocrono durante su ciclo. Plantas de origen de semillas de calidad superior producen más granos tanto en población de plantas como de forma aislada. El desarrollo de las plantas no fue un factor determinante para la productividad.

Palabras clave: Glycine max (L.) Merril; Vigor; Plasticidad; Suma térmica.

\section{Introdução}

A soja possui grande importância no Brasil e aumentos da produtividade e da produção desta cultura são registrados a cada ano (Cantarelli et al., 2015a), produtividades mais elevadas estão relacionadas aos genótipos com maior potencial produtivo e melhoria no manejo da cultura. Porém a evolução na produtividade cada vez está mais difícil exigindo mais esforços referentes às pesquisas quanto ao manejo e dos programas de melhoramento de soja (Perini et al., 2012).

A utilização de sementes com alto valor qualitativo tem despertado o interesse de vários pesquisadores fato que resultou em vários trabalhos sobre o assunto, em especial a cultura da soja. Os resultados dos trabalhos apontam que o uso de uma semente com boa qualidade fisiológica reflete positivamente na capacidade produtiva da cultura (Schuch et al., 2009; Silva et al., 2010, Bagateli et al., 2019; Ferrazza et al., 2020; Dias et al., 2021).

Sementes com baixo vigor apresentam menor porcentagem de emergência e consequentemente desuniformidade na formação do estande de plantas (Silva et al., 2013; Cantarelli et al., 2015b). Para compensar o uso destas sementes com baixa qualidade fisiológica ocorre o aumento da densidade de semeadura (Scheeren et al., 2010), mesmo assim pode ocorrer falha na distribuição de plantas. Assim há necessidade da planta compensar tais alhas, modiicando seus componentes de produção, esta capacidade de alteração nas características da planta é chamada de plasticidade.

Estudando a plasticidade como estratégia de redução da densidade de semeadura, Büchling, Oliveira Neto, Guerra e Bottega (2017) observaram que nas populações de 150, 250, 350 e 450 mil plantas ha ${ }^{-1}$ não apresentaram diferenças de desempenho, fato explicado pela alta capacidade de alteração na estrutura externa da planta ajustando características como número de ramificações, de nós reprodutivos, de vagens e de grãos.m². Já Mauad et al. (2010), observaram que a soja aumenta a quantidade de nós reprodutivos, vagens e grãos por vagem para compensar as baixas populações.

O desenvolvimento da soja soja pode ser indeterminado, determinado e semi-determinado, sendo as cultivares determinadas as mais cultivadas no Brasil (Procópio et al., 2013. Maior duração do período vegetativo tem sido observado para plantas com caracteristicas determinada, resultando em maior quantidade de nós por e altura das plantas no início do florescimento, entretanto, cultivares que apresentam crescimento indeterminado, interrompem seu crescimento somente no início da fase de enchimento de legumes, apesar de seu crescimento inicial ser mais lento que cultivares determinadas. Esta diferença genética entre cultivares requerem datações nas práticas de manejo (Zanon et al., 2015, 2016), com diferentes densidades populacionais e períodos de semeadura. 
Uma das recomendações observadas para a cultura da soja foi a redução da densidade de plantio que até aproximadamente 2010 foi de 40 plantas. $\mathrm{m}^{-2}$ para 30 plantas.m $\mathrm{m}^{-2}$ (RPSRS, 2012), até os dias atuais. Porém atualmente há uma população indicada para cada cultivar, definida pela obtentora conforme características genéticas, que pode ser de 20 ou até 18 plantas. $\mathrm{m}^{-2}$. O estande de plantas adequando por área é fundamental para que as plantas se desenvolvam e expressem seu potencial produtivo.

No entanto, populações de plantas baixas exigem sementes de elevada qualidade, pois cada planta vai responder pela produção de determinada quantidade de grãos. Quando a densidade desejada de plantas não é atingida, há necessidade de compensação pelas plantas presentes, porém estudo realizado por Fiss et al. (2018) constatou que o aumento da produção das plantas localizadas nas bordas de falhas não compensa a perda de produção causada pela lacuna nas linhas e Cantarelli et al. (2015a) que quanto menor o vigor, maior é a possibilidade de ocorrer falhas. Neste sentido o trabalho tem como objetivo avaliar o efeito da qualidade fisiológica das sementes na plasticidade da soja com crescimento determinado e indeterminado.

\section{Metodologia}

O trabalho desenvolvido foi um estudo de caso, conforme metodologia de estudo científico, proposta por Severino (2017). Foram instalados dois ensaios durante os períodos de 2015/2016 e 2016/2017, no munícipio de Ibirubá/RS (28³7'39'’S

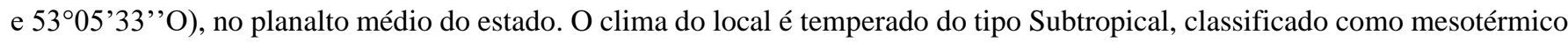
úmido, com chuvas distribuídos durante todo o ano, e com temperaturas elevadas no verão (Moreno, 1961). Solo de textura argilosa, bem drenados e interperizados, baixa ertilidade natural e altos teores de ferro, definido como LVd2 de acordo com Embrapa (2013).

Os tratamentos foram constituídos por sementes de soja com diferentes qualidades fisiológica (maior e menor) provenientes de quatro cultivares. A seleção das sementes foi realizada por apresentarem diferentes ciclos de desenvolvimento. Foram utilizadas as cultivares (BMX Ativa RR; FPS Urano RR; 5855 RSF IPRO; DM 5958 RSF IPRO). Os diferentes níveis de qualidade foi obtido por meio do envelhecimento de $25 \%$ das sementes do lote. Após, as sementes foram adicionadas ao restante das sementes do lote que não sofreu o envelhecimento, as quais foram homogeneizadas antes da semeadura.

A semeadura foi realizada através de um conjunto trator semeadora, em plantio direto, sobre restos culturas da cultura do Trigo. O espaçamento entre fileiras foi de $0,45 \mathrm{~m}$. Foi utilizado o delineamento inteiramente casualizado, tendo em vista a homogeneidade da área. As unidade experimentais, foram constituídas por fileiras com 10 metros de comprimento, sendo uma fileira de cultivo e duas de bordadura. No primeiro ano agrícola a data da semeadura foi em 19/11/2015 e no dia 30/11/2016 na segunda safra. Antes da semeadura, foi realizada a inoculação com Bradyrhizobium japonicum e o tratamento com piraclostrobina, tiofanato metílico e fipronil, na dose de $0,02 \mathrm{~mL}$ por quilograma de sementes. A posição da semente foi de 0,03 $\mathrm{m}$, na linha de semeadura, ajustadas através do limitador de profundidade da semeadora e houve um acompanhamento constante durante toda a semeadura afim de garantir essa uniformidade.

De acordo com a análise da fertilidade do solo, e objetivando atingir produtividade de 4 quilogramas por $\mathrm{m}^{2}$, foi definida a adubação de base, conforme Comissão de Fertilidade e Química do Solo - RS/SC (2016). Os manejos da cultura foram realizados na cultura conforme indicações técnicas da região. Controle fitossanitários foram realizados com a finalidade de manter o cultivo livre de fatores biótico que pudesse interferir no crescimento e desenvolvimento das plantas.

A identificação e classificação das plantas oriundas da maior e menor qualidade fisiológica, foi definida pelo intervalo de tempo para a emergência (Schuch et al., 2009). Considerou a emergência quando os cotilédones estavam totalmente visíveis, e completamente acima da superfície do solo. As plântulas que apresentaram um menor intervalo entre semeadura/plantio e emergência (5 dias), portanto, as que primeiro emergiam, foram consideradas plantas de origem de semente com maior vigor. Já as plântulas que apresentaram maior intervalo (7 dias) foram consideradas plantas de origem de semente com vigor menor. 
Posteriormente a emergência as plantas foram identificadas através de uma estaca. Em cada parcela identificou-se 8 plantas, sendo 4 de maior e outras 4 de menor vigor. Após identificação das plantas foi realizado o desbaste das plantas presentes até $0,4 \mathrm{~m}$ da linha de semeadura. Após a área foi conduzida sobre manejo fitossanitário como lavoura comercial até o final do seu ciclo.

A colheita foi realizada de forma manual, na qual as plantas foram identificadas e avaliadas quanto a produtividade por planta (PP), peso de cem grãos (PCG), grãos por planta (GP), grãos na haste principal (GH), legumes por planta (LP), grãos por ramos (GR), legumes por haste (LH), legumes nos ramos (LR), grãos por legumes (GL), grãos por legume na haste principal (GLH), grãos por legume nos ramos (GLR), conforme descrito por Decarli et al. (2021).

Os resultados foram avalidos quanto à análise de variância e os valores médios foram comparados pelo teste de Tukey a 5\% de significância pelo programa estatístico SISVAR (Ferreira, 2011).

\section{Resultados e Discussão}

Considerando o período em que o experimento foi conduzido a temperatura do ar (média diária) em 2015/16 (Figura 1A) e $2016 / 17$ (Figura 1B) foram de $23,2{ }^{\circ} \mathrm{C}$ e $23,0{ }^{\circ} \mathrm{C}$, respectivamente. Durante toda a estação de crescimento a temperatura do ar permaneceu acima da basal, que neste estudo foi considerado de $10{ }^{\circ} \mathrm{C}$, e ficou abaixo da temperatura ótima $\left(30{ }^{\circ} \mathrm{C}\right)$. Em 2015/2016 pode-se considerar que foi uma safra mais úmida do que a de 2016/17, totalizando um acumulado de precipitações de 832,2 mm em 2015/16 e 397 mm em 2016/17, no período de crescimento e desenvolvimento das folhas (Figuras 1A e 1B). Fato esse que pode ser explicado pela radiação solar e pelo déficit hídrico (Figuras 2A e 2B), pois mesmo sendo um ano chuvoso foi observado um déficit hídrico que abrangeu praticamente toda a fase reprodutiva da soja, sendo observada uma menor radiação solar em 2015/16 (Figura 1A e Figuras 2A e 2B). Já em 2016/17 houve um menor armazenamento de chuvas na fase de emissão de folhas, um período menor de restrição hídrica que começou uns cinco dias antes de 2015/16, mas que atingiu a fase de pleno florescimento e a fase de enchimento de grãos e uma maior radiação solar (Figura 1B e Figuras 2A e 2B). Em experimentos realizados em distintas condições ambientais, $80 \%$ da variação na produtividade é causada, em geral, pelo ambiente, enquanto apenas 10\% da variação ocorre em virtude dos efeitos de genótipos e da interação genótipo x ambiente (Asfaw et al., 2009; Meotti et al., 2012). 
Figura 1. Temperatura média diária $\left({ }^{\circ} \mathrm{C}\right)$ e precipitação $(\mathrm{mm})$ durante o ciclo da cultura da soja nos anos agrícolas $2015 / 2016$.

A

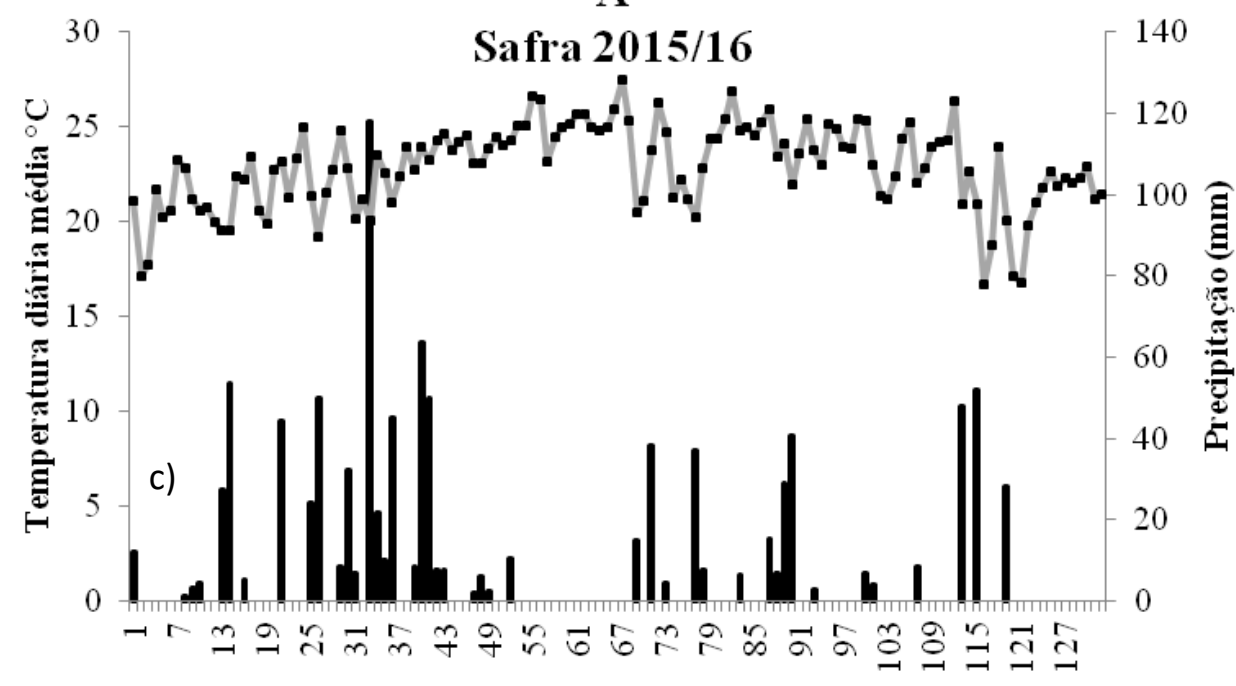

Dias após a semeadura

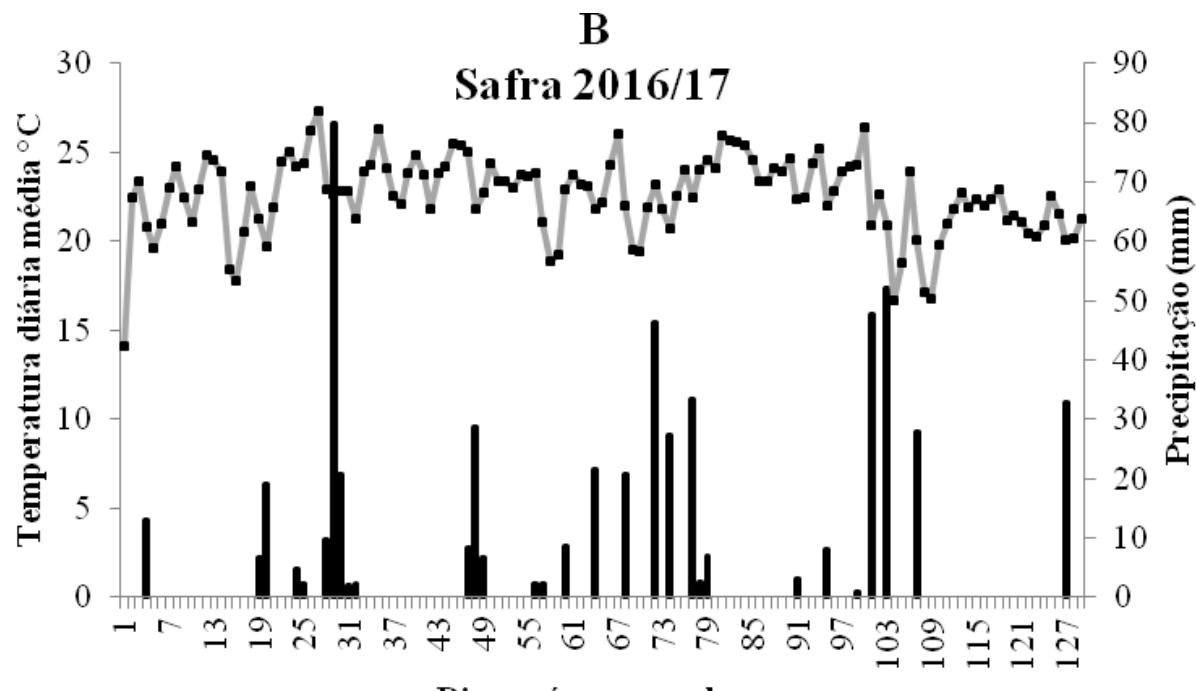

Dias após a semeadura

Fonte: Autores. 
Figura 2. Radiação solar $\left(\mathrm{kJm}^{2}\right)$ (A) e déficit hídrico $(\mathrm{mm})$ (B) durante o ciclo da cultura da soja nos anos agrícolas 2015/2016 e $2016 / 2017$.

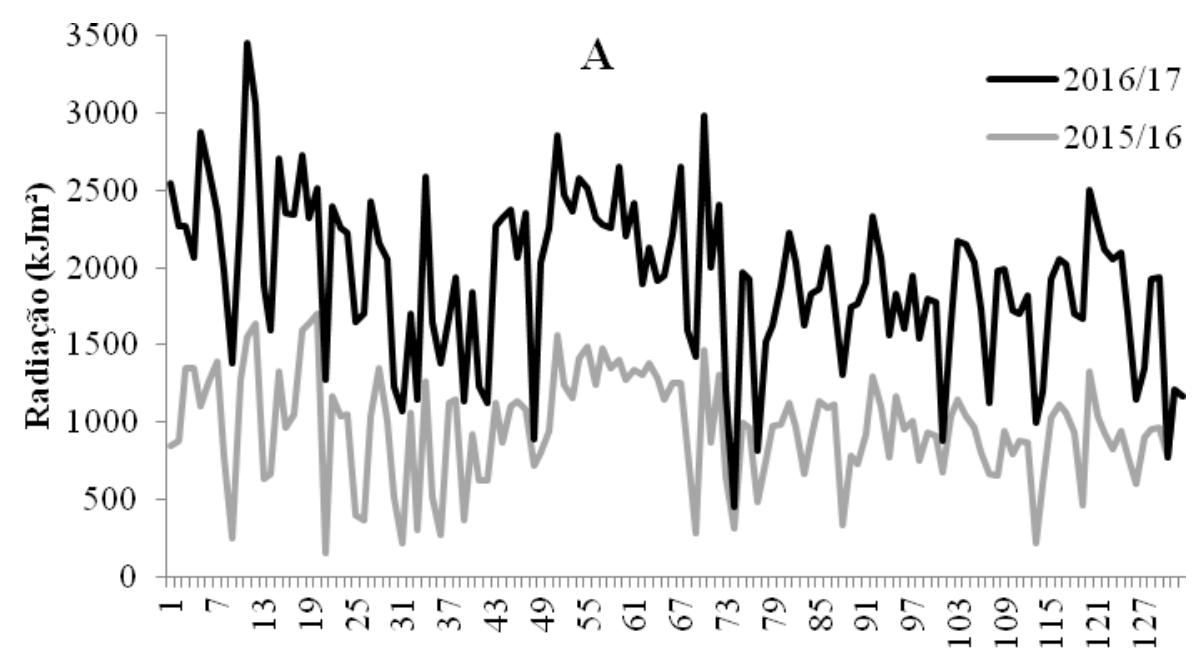

Dias após a semeadura

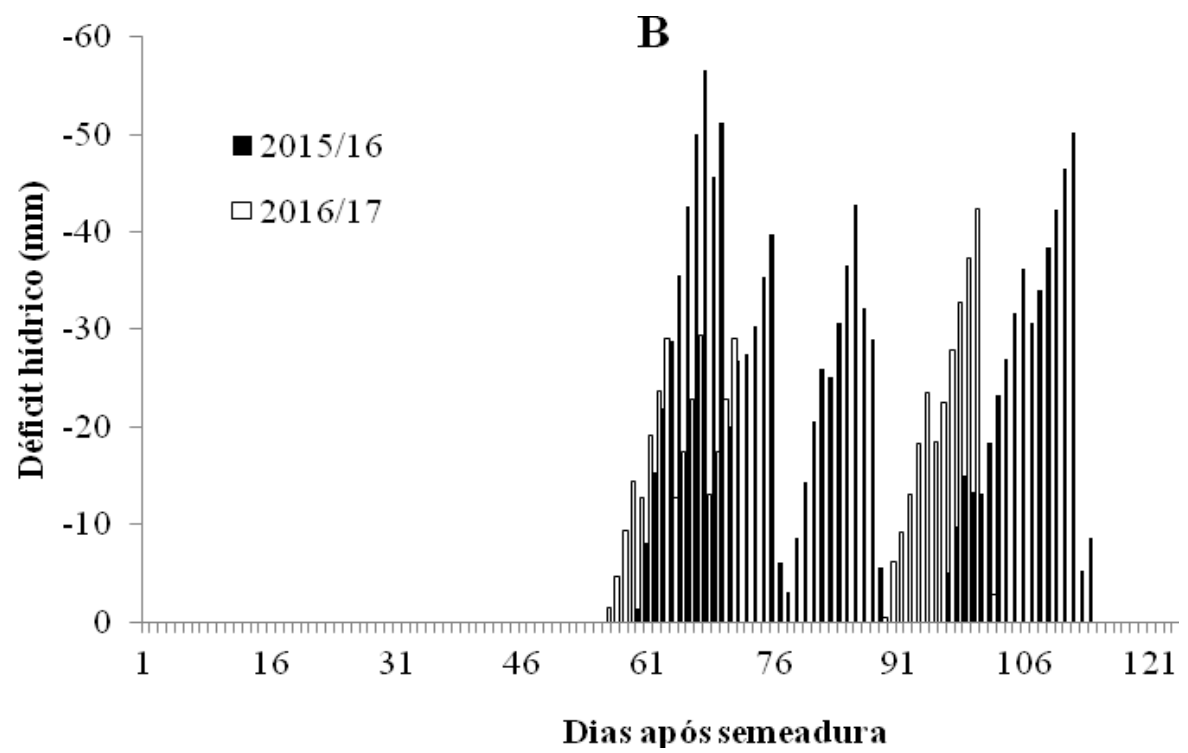

Fonte: Autores

Foi observado para qualidade diferença na produtividade por planta, grãos por planta, grãos na haste principal, grãos nos ramos, legumes por planta, legumes na haste principal e legumes nos ramos (Tabela 6). Utilizando como padrão uma população de plantas de 200.000 plantas/ha com uma produtividade por planta de $20,7 \mathrm{~g}$ em plantas de maior vigor teremos uma produção de $4140 \mathrm{~kg} / \mathrm{ha}$. Nessa mesma população com uma produtividade planta de $15 \mathrm{~g}$ (plantas com menor vigor) teremos uma produção de $3000 \mathrm{~kg} / \mathrm{ha}$. Se tivermos uma redução de $20 \%$ na população final (160.000 plantas/ha) com uma produção de 30 g planta obteremos um rendimento de $4800 \mathrm{~kg} / \mathrm{ha}$. Se observarmos os resultados obtidos (Tabela 1), mesmo em plantas isoladas onde não havia competição entre plantas por luz, água e nutrientes as plantas menos vigorosas não foram capazes de produzir $30 \mathrm{~g}$, tendo uma produção de 22,2 g planta resultando em produtividade inferior. Esse resultado demonstra a importância de se obter sementes com alto valor qualitativo. 
Tabela 1. Produtividade planta (PP) (g), peso de cem grãos (PCG), grãos planta-1 $(\mathrm{GP})$, grãos haste ${ }^{-1}(\mathrm{GH})$, grãos ramos ${ }^{-1}(\mathrm{GR})$, legumes planta ${ }^{-1}(\mathrm{LP})$, legumes haste ${ }^{-1}(\mathrm{LH})$, legumes ramos ${ }^{-1}(\mathrm{LR})$, grãos legumes ${ }^{-1}(\mathrm{GL})$, grãos legume haste ${ }^{-1}$ (GLH), grãos legume $\operatorname{ramos}^{-1}$ (GLR)de cultivares determinadas (BMX Ativa RR e FPS Urano RR) e indeterminadas (5855 RSF IPRO e Don Mario 5958 RSF IPRO) em dois níveis de vigor em plantas isoladas. Ibirubá, RS. Safra 2015/2016 e 2016/2017.

\begin{tabular}{|c|c|c|c|c|c|c|}
\hline & PP & PCG & GP & GH & GR & LP \\
\hline Maior & $39,1 \mathrm{a}$ & $15,7^{\mathrm{ns}}$ & $255,1 \mathrm{a}$ & $87,3 a$ & $167,8 \mathrm{a}$ & $115,3 \mathrm{a}$ \\
\hline Menor & $22,2 b$ & 16,3 & $142,3 b$ & $69,7 \mathrm{~b}$ & $72,6 b$ & $64,8 b$ \\
\hline BMX Ativa RR & $39,0 \mathrm{a}$ & $17,2 \mathrm{a}$ & $232,3 a$ & $76,4 a b$ & $155,9 a$ & $97,9^{\text {ns }}$ \\
\hline FPS Urano RR & $27,9 b$ & $17,3 \mathrm{a}$ & $172,3 b$ & $62,8 b$ & $109,5 b$ & 86,8 \\
\hline 5855 RSF IPRO & $30,7 \mathrm{ab}$ & $15,2 b$ & $205,8 \mathrm{ab}$ & $86,8 \mathrm{a}$ & $118,9 \mathrm{ab}$ & 94,8 \\
\hline DM 5958 RSF IPRO & $24,9 \mathrm{~b}$ & $14,3 \mathrm{~b}$ & $184,2 \mathrm{ab}$ & $88,0 \mathrm{a}$ & $96,3 b$ & 80,8 \\
\hline $2015 / 2016$ & $32,8^{\mathrm{ns}}$ & $17,7 \mathrm{a}$ & $194,6^{\text {ns }}$ & $67,0 \mathrm{~b}$ & $127,6^{\text {ns }}$ & $89,9^{\text {ns }}$ \\
\hline $2016 / 2017$ & 28,5 & $14,2 \mathrm{~b}$ & 202,7 & $90,0 \mathrm{a}$ & 112,7 & 90,2 \\
\hline Média & 30,6 & 16 & 198,7 & 78,5 & 120,2 & 90,1 \\
\hline \multirow[t]{2}{*}{ C.V. $(\%)$} & 29,31 & 7,08 & 26,76 & 21,1 & 35,42 & 25,24 \\
\hline & LH & LR & & GL & GLH & GLR \\
\hline Maior & $38,6 a$ & $76,7 \mathrm{a}$ & & $2,2^{\mathrm{ns}}$ & $2,2^{\mathrm{ns}}$ & $2,1^{\mathrm{ns}}$ \\
\hline Menor & $31,5 b$ & $33,3 b$ & & 2,2 & 2,2 & 2,2 \\
\hline BMX Ativa RR & $33,0 \mathrm{ab}$ & $64,9 \mathrm{a}$ & & $2,3 a$ & $2,3 \mathrm{a}$ & $2,3 a$ \\
\hline FPS Urano RR & $30,9 b$ & $55,8 \mathrm{ab}$ & & $2,0 \mathrm{~b}$ & $2,0 \mathrm{~b}$ & $1,9 b$ \\
\hline 5855 RSF IPRO & $37,8 \mathrm{a}$ & $57,0 \mathrm{ab}$ & & $2,2 \mathrm{a}$ & $2,3 \mathrm{a}$ & $2,1 \mathrm{ab}$ \\
\hline DM 5958 RSF IPRO & $38,4 \mathrm{a}$ & $42,4 \mathrm{~b}$ & & $2,3 \mathrm{a}$ & 2,2ab & $2,3 \mathrm{a}$ \\
\hline $2015 / 2016$ & $30,9 \mathrm{~b}$ & $59,0^{\text {ns }}$ & & $2,1^{\mathrm{ns}}$ & $2,1 \mathrm{~b}$ & $2,2^{\text {ns }}$ \\
\hline $2016 / 2017$ & $39,2 \mathrm{a}$ & 51 & & 2,2 & $2,3 \mathrm{a}$ & 2,1 \\
\hline Média & 35 & 55 & & 2,2 & 2,2 & 2,2 \\
\hline C.V. (\%) & 19,43 & 35,01 & & 9,9 & 10,94 & 13,62 \\
\hline
\end{tabular}

* Médias seguidas pela mesma letra minúscula coluna maiúscula linha não diferem entre si pelo teste de Tukey a 5\% de probabilidade de erro. ns Não significativo a 5\% de probabilidade de erro. Fonte: Autores.

Em plantas isoladas observou-se interação significativa para qualidade e ano nas variáveis produtividade obtida por planta, legumes por planta, legumes nos ramos, grãos por planta e grãos nos ramos (Tabela 2). Sendo que as plantas oriundas de sementes com maior vigor apresentaram maior produção nas duas safras avaliadas em relação às de baixo vigor. Esse resultado se deve as demais variáveis que foram superiores sempre nas plantas de qualidade superior em relação às de qualidade inferior. Onde nas plantas com maior vigor na safra 2015/16 foi superior a safra 2016/17 com 44,6 g e 33,6 g, respectivamente. Nas plantas com menor vigor não houve diferença entre os períodos avaliados. 
Tabela 2. Produtividade planta ${ }^{-1}(\mathrm{PP})(\mathrm{g})$, legumes planta ${ }^{-1}(\mathrm{LP})$, legumes $\operatorname{ramos}^{-1}(\mathrm{LR})$, grãos planta ${ }^{-1}(\mathrm{GP})$ e grãos ramos ${ }^{-1}(\mathrm{GR})$, de cultivares determinadas (BMX Ativa RR e FPS Urano RR) e indeterminadas (5855 RSF IPRO e Don Mario 5958 RSF IPRO) em dois níveis de vigor em plantas isoladas. Ibirubá, RS. Safra 2015/2016 e 2016/2017.

\begin{tabular}{|c|c|c|c|c|c|c|}
\hline & \multicolumn{2}{|c|}{--------------PP---------- } & \multicolumn{2}{|c|}{----------------LP---------------- } & \multicolumn{2}{|c|}{--------------LR-------------- } \\
\hline & $2015 / 2016$ & $2016 / 2017$ & $2015 / 2016$ & $2016 / 2017$ & $2015 / 2016$ & $2016 / 2017$ \\
\hline Maior & 44,6 aA & $33,6 \mathrm{aB}$ & 123,2 a $\mathrm{A}$ & $107,4 \mathrm{aA}$ & $87,7 \mathrm{aA}$ & $65,7 \mathrm{aB}$ \\
\hline Menor & $21,0 \mathrm{bA}$ & $23,4 \mathrm{bA}$ & $56,5 \mathrm{bB}$ & $73,0 \mathrm{bA}$ & $30,3 \mathrm{bA}$ & $36,4 \mathrm{bA}$ \\
\hline Média & \multicolumn{2}{|c|}{30,6} & \multicolumn{2}{|c|}{90,1} & \multicolumn{2}{|c|}{55} \\
\hline C.V. $(\%)$ & \multicolumn{2}{|c|}{29,31} & \multicolumn{2}{|c|}{25,24} & \multicolumn{2}{|c|}{35,01} \\
\hline \multicolumn{7}{|c|}{ - } \\
\hline & \multicolumn{2}{|c|}{$2015 / 2016$} & $2016 / 2017$ & $2015 / 2016$ & \multicolumn{2}{|c|}{$2016 / 2017$} \\
\hline Maior & \multicolumn{2}{|l|}{$265,0 \mathrm{aA}$} & $245,1 \mathrm{aA}$ & $187,4 \mathrm{aA}$ & \multicolumn{2}{|c|}{$148,1 \mathrm{aB}$} \\
\hline Menor & \multicolumn{2}{|l|}{$124,2 \mathrm{bA}$} & $160,3 \mathrm{bA}$ & $67,8 \mathrm{bA}$ & \multicolumn{2}{|c|}{$77,4 \mathrm{bA}$} \\
\hline Média & \multicolumn{3}{|c|}{198,7} & & \multicolumn{2}{|l|}{120,2} \\
\hline C.V. $(\%)$ & \multicolumn{3}{|c|}{26,76} & & \multicolumn{2}{|l|}{35,42} \\
\hline
\end{tabular}

* Médias seguidas pela mesma letra minúscula coluna maiúscula linha não diferem entre si pelo teste de Tukey a 5\% de probabilidade de erro. Fonte: Autores.

Na variável legumes por planta (Tabela 2) em plantas de menor qualidade foi obtido um maior número de legumes na safra 2016/17 com 73 em relação a 2015/16 com 56,5. Enquanto, na variável legumes nos ramos e grãos nos ramos não houve diferença entre os períodos. Já para as de maior qualidade constatou-se um maior número de legumes e grãos nos ramos na safra 2015/16 com 87,7 e 187,4, respectivamente. Schuch et al. (2009) concluiram que o rendimento obtido em plantas de soja com cultivo isolado quando se utilizou sementes de alto vigor foi de aproximadamente, $25 \%$ superior em relação às de baixa e que o maior rendimento foi determinado pelo componente número de vagens por planta.

Para o peso de cem grãos (g) e produtividade planta (g) em plantas isoladas foi verificada interação significativa (Tabela 3). Sendo que, para o peso de cem grãos, a interação cultivar e ano, observa-se que para o ano 2015/16 os maiores pesos de grãos foram obtidos para BMX Ativa RR e 5855 RSF IPRO com 19,1 g e 20,2 g respectivamente. Já em $2016 / 17$ a BMX Ativa RR teve o maior peso de grãos (15,2 g), mas não diferiu da FPS Urano RR com 13,8 g e 5855 RSF IPRO com 14,4 g. Na interação observou-se na safra 2015/16 todas as cultivares apresentaram um peso de grãos maior que na safra 2016/17. Isso se deve a distribuição das chuvas durante a condução da soja (Figura 1A) e ao período de déficit hídrico (Figura 2B). 
Tabela 3. Peso de 100 grãos (g) e produtividade planta $^{-1}$ (g) de cultivares determinadas (BMX Ativa RR e FPS Urano RR) e indeterminadas (5855 RSF IPRO e Don Mario 5958 RSF IPRO) em dois níveis de vigor em plantas isoladas. Ibirubá, RS. Safra 2015/2016 e 2016/2017.

\begin{tabular}{ccccc}
\hline & \multicolumn{2}{c}{ Peso de 100 Grãos (g) } & \multicolumn{2}{c}{ Produtividade Planta (g) } \\
\hline & $2015 / 2016$ & $2016 / 2017$ & Maior & Menor \\
\hline BMX Ativa RR & $19,1 \mathrm{aA}$ & $15,2 \mathrm{aB}$ & $52,7 \mathrm{aA}$ & $25,3 \mathrm{aB}$ \\
FPS Urano RR & $16,5 \mathrm{bA}$ & $13,8 \mathrm{abB}$ & $35,0 \mathrm{bA}$ & $20,9 \mathrm{aB}$ \\
5855 RSF IPRO & $20,2 \mathrm{aA}$ & $14,4 \mathrm{abB}$ & $39,5 \mathrm{bA}$ & $22,0 \mathrm{aB}$ \\
DM 5958 RSF IPRO & $15,1 \mathrm{bA}$ & $13,4 \mathrm{bB}$ & $29,2 \mathrm{bA}$ & 30,6 \\
\hline Média & & 16 & & 29,31 \\
C.V. $(\%)$ & 7,08 & & \\
\hline
\end{tabular}

* Médias seguidas pela mesma letra minúscula coluna maiúscula linha não diferem entre si pelo teste de Tukey a 5\% de probabilidade de erro. Fonte: Autores.

Quando trabalhado com produtividade por planta (g) observou-se interação significativa para cultivar e qualidade (Tabela 3). Nas plantas com qualidade superior constatou-se que a cultivar mais produtiva foi a BMX Ativa RR com 52,7 g planta. Já nas plantas de menor qualidade as cultivares não apresentaram diferenças entre si. Na interação, a BMX Ativa RR, FPS Urano RR e 5855 RSF IPRO obtiveram uma maior produtividade por planta quando oriundas de sementes de maior qualidade, fato este não observado para a cultivar Don Mario 5958 IPRO, que não apresentou diferença estatística para a qualidade de sementes.

\section{Conclusão}

O plastocrono foi influenciado pela disponibilidade hídrica, e o tipo de crescimento não foi determinante na definição do plastocrono. Plantas provenientes de sementes de baixo vigor necessitam de maior plastocrono durante seu ciclo.

Sementes de maior qualidade originam plantas que produzem mais grãos tanto em população de plantas como de forma isolada. O tipo de crescimento não foi fator determinante para a variável produtividade.

Sugere-se para trabalhos futuros, ampliar os estudos com outras variedades de soja verificando a qualidade de sementes no plastocrono e produtividade.

\section{Referências}

Asfaw, A., Alemayehu, F., Gurum, F., \& Atnaf, M. (2009). AMMI and SREG GGE biplot analysis for matching varieties onto soybean production environments in Ethiopia. Scientific Research and Essay, 4(11), 1322-1330.

Bagateli, J. R., Dorr, C. S., Schuch, L. O. B., \& Meneghello, G. E. (2019). Productive performance of soybean plants originated from seed lots with increasing vigor levels. Journal of Seed Science, 41(2), 151-159.

Büchling, C., Oliveira Neto, A. M., Guerra, N., \& Bottega, E. L. (2017). Uso da plasticidade morfológica como estratégia para a redução da população de plantas em cultivares de soja. Revista Agrarian, 10(35), 22-30. doi: 10.30612/agrarian.v10i35.3931

Cantarelli, L. D., Schuch, L. O. B., Rufino, C. A., Tavares, L. C., \& Vieira, J. F. (2015b). Physiological seeds quality: spatial distribution and variability among soybean plant population. Bioscience Journal, 31(2), 344-351.

Cantarelli, L. D., Schuch, L. O. B., Tavares, L. C., \& Rufino, C. A. (2015a). Variabilidade de plantas de soja originadas de sementes de diferentes níveis de qualidade fisiológica. Acta Agronómica, 64(3), 234-238.

Decarli, L., Ludwig, M. P., Decarli, J., Garbin, T., \& Martins, J. D. (2021). Plastocrono e produtividade de soja com diferentes hábitos de crescimento em função da qualidade das sementes. Brazilian Journal of Development, 7(4), 39163-39179.

Dias, G. H. O., Lisboa, L. A. M., Ferreira, J. P. D. S., \& Rocha, E. A. (2021). Desenvolvimento de cultivares de soja de crescimento indeterminado após a poda apical. Research, Society and Development, 10(5), e46510513688. doi: 10.33448/rsd-v10i5.13688 
Research, Society and Development, v. 11, n. 3, e47311326555, 2022

(CC BY 4.0) | ISSN 2525-3409 | DOI: http://dx.doi.org/10.33448/rsd-v11i3.26555

Embrapa. (2013). Centro Nacional de Pesquisa de Solo (3 ed. revista e ampliada, 353p). Embrapa.

Ferrazza, F. L. F., Jacoboski, D. T. K., Wyrepkowski, A., Rodrigues, L., Figueiro, A. G., Paraginski, R. T. Qualidade de sementes e parâmetros produtivos de sementes de soja submetidas a diferentes tratamentos de sementes antes da semeadura. Research, Society and Development, 9(9), e47996232. doi: 10.33448/rsdv9i9.6232

Ferreira, D. F. (2011). Sisvar: a computer analysis system. Ciência e Agrotecnologia, 35(6), 1039-1042.

Fiss, G., Schuch, L. S. O., Peske, S. T., Castellanos, C. I. S., Meneghello, G. M., \& Aumonde, T. Z. (2018). Produtividade e características agronômicas da soja em função de falhas na semeadura. Revista de Ciências Agrárias, 61, 1-7. doi: 10.22491/rca.2018.2477

Mauad, M., Silva, T. L. B., Almeida Neto, A. I., \& Abreu, V. G. (2010). Influência da densidade de semeadura sobre características agronômicas na cultura da soja. Revista Agrarian, 3(9), 175-181.

Meotti, G. V., Benin, G., Silva, R. R., Beche, E., \& Munaro, L. B. (2012). Épocas de semeadura e desempenho agronômico de cultivares de soja. Pesquisa Agropecuária Brasileira, 47(1), 14-21.

Moreno, J. A. (1961). Clima do Rio Grande do Sul. Porto Alegre: Secretaria da Agricultura, Diretoria de Terras e Colonização, Secção de Geografia.

Perini, L. J., Fonseca, N. S. J., Destro, D., \& Prete, C. E. C. (2012). Componentes da produção em cultivares de soja com crescimento determinado e indeterminado. Semina: Ciências Agrárias, 33(suplemento 1), 2531-2544.

Procópio, S. O., Balbinot Júnior, A. A., Debiasi, H., Franchini, J. C., \& Panison, F. (2013). Plantio cruzado na cultura da soja utilizando uma cultivar de hábito de crescimento indeterminado. Amazonian Journal, 56, 319-325.

RPSRS. (2012). Indicações técnicas para a cultura da soja no Rio Grande do Sul e em Santa Catarina, safras 2012/2013 e 2013/2014. In XXXIX Reunião de Pesquisa de Soja da Região Sul, Passo Fundo, RS.

Scheeren, B. R., Peske, S. T., Schuch, L. O. B., \& Barros, A. C. A. (2010). Qualidade fisiológica e produtividade de sementes de soja Revista Brasileira de Sementes, 32(3), 35-41.

Schuch, L. O., Kolchinski, E. M., \& Finato, J. A. (2009). Qualidade fisiológica da semente e desempenho de plantas isoladas em soja. Revista Brasileira de Sementes, 31(1), 144-149.

Severino, A. J. (2017). Metodologia do trabalho científico. Cortez.

Silva, C. S., Schuch, L. O. B., Olivo, M., \& Seus, R. (2013). Desempenho de plantas isoladas de soja, biometria e qualidade fisiológica das sementes. Revista da FZVA Uruguaiana, 19(1), 1-9.

Silva, J. B., Lazarini, E., \& Sá, M. E. (2010). Comportamento de sementes de cultivares de soja, submetidos a diferentes períodos de envelhecimento acelerado. Bioscience Journal, 26(5), 755-762.

Zanon, A. J., Streck, N. A., Rocha, T. S. M., Alberto, C. M., Bartz, A. C., Paula, G. M. \& Bexaira, K. P. (2016). Efeito do tipo de crescimento no desenvolvimento de cultivares modernas de soja após o início do florescimento no Rio Grande do Sul. Bragantia, 75(4), 446-458.

Zanon, A. J., Winck, J. E. M., Streck, N. A., Rocha, T. S. M., Cera, J. C., Richter, G. L. \& Marchesan, E. (2015). Desenvolvimento de cultivares de soja em função do grupo de maturação e tipo de crescimento em terras altas e terras baixas. Bragantia, 74(4), 400-411. 